\section{Glittering prizes}

Joseph Silk

\section{A Hundred Billion Stars.}

By Mario Rigutti. Translated by Mirella Giacconi.

MIT Press: 1984. Pp. 285. \$25,

£26.15.

To ALL Earth-bound observers, stars are brilliant points of light, unresolved even by our largest telescopes; poets and astronomers alike must work with similar raw material. Imagination aids the poet in conjuring up alien vistas, while the astronomer is assisted by sophisticated devices that split the light into a thousand frequencies, unravelling the most intimate nature of the stellar matter. It is a rare astronomer who is capable of clearly communicating his esoteric discoveries, his knowledge of what really constitutes a star, to the uninitiated audience; and, more, of enthralling his readers, who are too frequently abused by sycophantic disciples of pseudo-science.

In One Hundred Billion Stars, Mario Rigutti has escaped from the dreary level of popular science writing that permeates the pages of numerous magazines. He does not give us breathless prose, studded with superlatives, yet manages to transmit a genuine awe for the starry heavens. Professor Rigutti's style is chatty and

Advertisement

\section{ANSW涢S}

to your questions about nuclear energy



answers and facts

about nuclear.

AMERICAN NUCLEAR SOCIETY

555 N. Kensington Ave.

La Grange Park, IL 60525

(312) 352-6611 informal. He writes as though he were telling a bedtime story to a child, and succeeds in captivating people of all ages. He takes occasional potshots outside the world of astronomy, rightly believing that practising scientists cannot ignore social and political issues. His unassuming approach and his evident sincerity provide a refreshing perspective on the world of the stars. Due credit should go to the translator, Mirella Giacconi, who appears to have ably succeeded in capturing the spirit of the original Italian text.

The book commences with a discussion of the Sun, a logical place to begin. It continues with the inner planets, and then

\section{Pi in the sky}

\section{John D. Barrow}

Constructing the Universe.

By David Layzer.

Scientific American Library: 1984.

Pp.313. \$21.95, 115.95. (Members of the Library only.)

Stephen Hawking's Universe.

By John Boslough.

William Morrow: 1984. Pp.158. \$12.95.

DAVID Layzer's addition to the Scientific American Library is extremely ambitious. It aims to tell the story of cosmological reasoning from the pre-Socratics to the present day. Not that it is just a history book: the author's approach is distinguished by his inclusion of accounts of the mathematical reasoning used by the past masters in their classic investigations. In this way one is able to see the actual logic of scientific development, a far better approach than the stream-lined résumé which uses modern concepts to tidy things up retrospectively. Layzer also enlivens the story by commenting on the discussion and dissent that surrounded the introduction of new concepts; for example, Berkeley's attitude to "fluxions". These early chapters dealing with historical developments are carefully written, beautifully illustrated and well-integrated into a discussion of the scientific method which runs through the text.

The next part of the book provides a well devised description of special relativity and a brief survey of the key concepts involved in the formulation, verification and application of general relativity. With all this groundwork laid out so thoroughly I expected to find as its climax a superb final portion of the book devoted to modern cosmology. I was disappointed.

The author makes much of the importance of correctly formulating the Cosmological Principle but then does so in a misleading and unappealing way by defining it to mean that "the universe itself has the same spatial symmetries as the laws that govern its structure and evolution". This statement is said to be "axiomatic", comes the major section, on our galaxy. Most of what is known about our galaxy is told, not too much progress having been made in stellar evolution since 1978 when the Italian edition appeared. The new discoveries have certainly not changed our overall view of our local celestial environment, and the book does not suffer too adversely from this shortcoming. The author rightly concentrates on capturing the essence of the underlying truth behind those glittering points of light in the sky, and, by and large, he succeeds.

Joseph Silk is Professor of Astronomy at the University of California, Berkeley.

which is unfortunate, since it is clearly false. Furthermore, the claim that any statistical formulation of the Cosmological Principle must be rejected because it implies preferred places is also illogical. We can only make scientific statements about the observable portion of the Universe and there may well exist "preferred" places where observers are most likely to be found. The "many-bubble" inflationary universe models considered recently need not satisfy any "axiomatic" cosmological principle.

However, my main worry about the book arises from the author's cursory, and to my mind, dishonest treatment of the observational evidence for the hot big bang model - a topic which ought to have been extensively and fairly discussed in a book such as this. The author displays "predictions" of the cosmic abundances of helium and deuterium from the hot big bang model supposedly made in 1965 and 1984 , along with the "observations" of these abundances at those same dates. Virtually all the information provided is incorrect. The presently observed cosmic helium mass fraction does not lie in the range $10-21 \%$ as claimed, nor did it lie in the range $26-28 \%$ in 1965 . As far as I am aware, there were no big bang deuterium predictions in 1965 , nor were there any extraterrestrial measurements. Nor are the predictions any different, in principle, over this time span; they are only made to look very different by the author's assumption that the present baryon density must be ten times higher than we believed in 1965 because of the flat rotation curves of spiral galaxies. But if the dark matter responsible for that state of affairs is non-baryonic, it does not deplete the deuterium abundance in the way Layzer claims. Further, quite a lot of significance is attributed to the Woody-Richards measurement of a distortion in the microwave background even though its statistical significance has since been downgraded.

Having presented this brief caricature of the hot big bang model, the author then devotes 22 pages to detailed exposition of his own speculative ideas developed in a student's thesis in 1973. These ideas regarding a non-primordial origin of the 